\title{
Allelic sequence heterozygosity in single Giardia parasites
}

\author{
Johan Ankarklev ${ }^{1 *}$, Staffan G Svärd ${ }^{1}$ and Marianne Lebbad²
}

\begin{abstract}
Background: Genetic heterogeneity has become a major inconvenience in the genotyping and molecular epidemiology of the intestinal protozoan parasite Giardia intestinalis, in particular for the major human infecting genotype, assemblage B. Sequence-based genotyping of assemblage B Giardia from patient fecal samples, where one or several of the commonly used genotyping loci (beta-giardin, triosephosphate isomerase and glutamate dehydrogenase) are implemented, is often hampered due to the presence of sequence heterogeneity in the sequencing chromatograms. This can be due to allelic sequence heterozygosity (ASH) and /or co-infections with parasites of different assemblage B sub-genotypes. Thus, two important questions have arisen; i) does ASH occur at the single cell level, and/or ii) do multiple sub-genotype infections commonly occur in patients infected with assemblage B, G. intestinalis isolates?

Results: We used micromanipulation in order to isolate single Giardia intestinalis, assemblage B trophozoites (GS isolate) and cysts from human patients. Molecular analysis at the tpi loci of trophozoites from the GS lineage indicated that ASH is present at the single cell level. Analyses of assemblage B Giardia cysts from clinical samples at the $b g$ and tpi loci also indicated ASH at the single cell level. Additionally, alignment of sequence data from several different cysts that originated from the same patient yielded different sequence patterns, thus suggesting the presence of multiple sub-assemblage infections in congruence with ASH within the same patient.

Conclusions: Our results conclusively show that ASH does occur at the single cell level in assemblage B Giardia. Furthermore, sequence heterogeneity generated during sequence-based genotyping of assemblage $B$ isolates may possess the complexity of single cell ASH in concurrence with co-infections of different assemblage B subgenotypes. These findings explain the high abundance of sequence heterogeneity commonly found when performing sequence based genotyping of assemblage B Giardia, and illuminates the necessity of developing new G. intestinalis genotyping tools.
\end{abstract}

\section{Background}

The globally occurring diarrhea-causing protozoan, Giardia intestinalis (syn. G. lamblia and G. duodenalis), makes up a species complex of eight different genotypes or assemblages, A-H [1], where assemblages A and B can cause disease in humans [2]. Understanding of the epidemiology of the disease caused by G. intestinalis (giardiasis) has been hampered due to the genomic complexity of the parasite (cellular ploidy of $4 \mathrm{~N}-16 \mathrm{~N}$ in two nuclei) [3], along with the genetic heterogeneity that is present in assemblage B Giardia isolates [4-6]. The most

\footnotetext{
* Correspondence: johan.ankarklev@icm.uu.se

'Department of Cell and Molecular Biology, BMC, Uppsala University,

Husargatan 3, Box 596, Uppsala SE-751 24, Sweden

Full list of author information is available at the end of the article
}

commonly used genotyping loci; beta-giardin, glutamate dehydrogenase and triose- phosphate isomerase $(b g, g d h$ and $t p i$, respectively) have low discriminatory power when applied to assemblage A Giardia. Assemblage A subassemblages may only be discriminated at a few positions, due to a high level of conservation in these genes in assemblage A isolates, however, three different subassemblages have been established at the current loci, namely AI, AII and AIII. In assemblage B on the contrary, high variability in the form of mixed base polymorphisms has been observed at these loci, which has impeded proper epidemiological analyses [7-11]. It is not known if this genetic heterogeneity is due to high allelic sequence heterozygosity (ASH) in assemblage B parasites [12] and/ or due to common mixed infections with different sub-

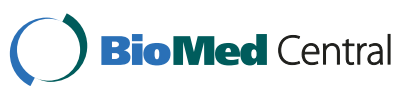


groups of assemblage B Giardia [5]. Within-species diversity has recently gained increased recognition and has been reported in pathogenic bacteria, fungi as well as in other protozoan parasites such as Plasmodium falciparum [1315]. It has been demonstrated that both polyclonal (infection by phylogenetically divergent clones) and monoclonal (infection by members of a single clone that display microheterogeneity) diversity exists in patients with single species infections [13]. This phenomenon is commonly seen in patients harboring chronic infections, which is, interestingly a common problem in giardiasis patients [2] To date no attempts have been made in investigating whether the occurrence of ASH in sequences generated from clinical assemblage B Giardia samples, commonly originate from a single isolate or a mosaic of different isolates. Single cell analyses would be required to resolve this issue. However, isolation of single Giardia trophozoites from culture or cysts from clinical Giardia samples for the purpose of direct comparative sequence analyses without in vitro growth has not previously been performed to the best of our knowledge. Previous methods that have been utilized for the purpose of cloning Giardia parasites are labor intensive and do not guarantee the establishment of single cells for molecular analyses [16-19].

Micromanipulation with size-specific micro-capillaries allows very sensitive discrimination, where single cells from a diluted fecal sample can be detected against a background, singled out, and transferred to a pure drop of liquid for re-verification of the clonality of the cell before proceeding to downstream analyses. In the malaria research field, micromanipulation has been applied for qualitative isolation of specific cells from a suspension of mixed cell types and mixed phenotypes, i.e. isolation of $P$. falciparum infected red blood cells (iRBCs) from a rosetting cluster for molecular analyses [20] or the isolation of P. falciparum iRBCs at a certain stage in the cell cycle, for molecular analyses [21]. In Giardia this approach has been used to isolate single cells for further growth in vitro and isoenzyme analysis of the cloned population [17]. The aim of our work was to use micromanipulation to efficiently isolate and sequence single Giardia assemblage B trophozoites grown in vitro, and single cysts isolated from human giardiasis patients, in order to properly verify genetic heterogeneity on the single cell level without growth in vitro. This approach can assess whether genetic heterogeneity identified in clinical assemblage B isolates is due to $\mathrm{ASH}$, mixed sub-assemblage infection or a combination of the two.

\section{Methods}

\section{Cell lines and clinical samples}

Giardia intestinalis GS/M (H7), assemblage B, was cultured in TYI-S-33 at optimal growth conditions [12] and seeded twice weekly prior to single cell analysis. Clinical
G. intestinalis samples were obtained from patients enrolled in an epidemiology study involving more than 200 giardiasis patients at the Karolinska University Hospital, the Department of Communicable Disease Control and Prevention, Stockholm County Council, and the Swedish Institute for Communicable Disease Control [8]. The Regional Ethics Committee of Karolinska Institutet, Stockholm, Sweden, has approved usage of the clinical samples. Crude DNA from all isolates were subject to PCR and subsequent sequencing of the $b g$, tpi, and $g d h$ loci and samples used in this study were evaluated based on several stringent criteria; 1) samples had to include assemblage B G. intestinalis cysts, 2) cyst load in the patient fecal samples had to exceed 100 cysts per $10 \mu$ l concentrated fecal suspension, 3) DAPI stained samples had to yield $>80 \%$ cysts with intact DNA in the nuclei, 4) sequences generated from multi-locus genotyping (MLG) of the samples had to indicate double peaks in the chromatograms at several positions on one or several of the genotyping loci used in the previous study. Three patient samples were finally included in the study, Sweh197 and Sweh212 which both included assemblage B Giardia, and Sweh207, which included a mixed assemblage A and B infection. The patients had prior to infection visited Iraq (Sweh197), Brazil (Sweh212), and India (Sweh207) [8].

\section{Purification of cysts from fecal samples}

Fresh fecal samples were examined on wet smears using light microscopy, and stored at $4^{\circ} \mathrm{C}$ prior to extraction of DNA or purification of cysts. FITC labeled CWP (cystwall protein) -specific antibodies (Agua-Glo, Waterborne Inc., New Orleans, LA, USA) and counterstaining with DAPI (4'6-diamino-2-phenyl-indole) were utilized to evaluate the level of viable cysts in each crude patient sample. Cysts were purified from fecal material using a density gradient centrifugation as earlier described [5].

\section{Isolation of single Giardia cysts and trophozoites}

Single, Giardia cysts (Sweh197, Sweh 207 and Sweh 212) and trophozoites (GS/M H7) were isolated according to a previously described methodology [20] with slight alterations. In brief, micromanipulation was performed on diluted and purified cysts from patient fecal samples, as well as chilled diluted Giardia trophozoites from cell cultures, using the MN-188 (Narishige, Tokyo, Japan) micromanipulator with sterile micropipettes, and an inverted Nikon Diaphot 300 microscope (Nikon, Tokyo, Japan) (Additional file 1). The sterile pipettes were synthesized "in house" using the P-97 pipette puller (Sutter Instruments, Novato, CA, US) and internal diameters varied from $6 \mu \mathrm{m}$ to $8 \mu \mathrm{m}$ based on the differences in size and outer membrane rigidity between the Giardia trophozoites and cysts. Prior to micromanipulation, all 
isolates were diluted down to a working concentration of approximately $10-20$ cells per $1 \mu$ l solution. Picked cells were transferred to a $2 \mu \mathrm{l}$ drop of 1XPBS (trophozoites) or $\mathrm{ddH}_{2} \mathrm{O}$ (cysts) and viewed under the microscope for additional verification of proper isolation of single cells. Cells were subsequently transferred to PCR tubes using a micropipette.

A total of 44 Giardia cysts from patient samples that had previously been labeled with FITC labeled CWPspecific antibodies were picked and placed on a 12 well microscope slide (Thermo Fisher Scientific, Sweden) in order to verify the specificity of the single cell isolation method. Isolated single cysts, were evaluated by trained, independent microscopists using a Nikon Eclipse E400 Fluorescent microscope (Nikon, Tokyo, Japan). In addition, one of the wells of the slides was always used as a negative control, here liquid was transferred from the fecal suspension onto one of the wells on the 12-well slides and analyzed. Such negative controls were also implemented in the PCR based assays.

\section{DNA extraction of single Giardia cysts and trophozoites}

Two different methods were evaluated for efficient extraction of DNA from single Giardia trophozoites in order to establish a sensitive enough method for the purpose of generating sequences from single cells, where ASH can properly be assessed; 1 . Snap freezing/thawing of single trophozoites in $1 \mathrm{XPBS}$ at $-80^{\circ} \mathrm{C}$ post-isolation. 2. DNA extraction of single trophozoites using DNAreleasy (NIPPON Genetics Europe, No LS02, Düren, Germany). Isolated single Giardia trophozoites were deposited in $2 \mu \mathrm{l}$ drops of 1XPBS, transferred to PCR tubes containing $3 \mu \mathrm{l}$ DNAreleasy and treated according to the manufacturer's instructions (both the short and the long protocols provided by the manufacturer were assayed for extraction of DNA from single cysts). Subsequently, PCR reaction mixtures were added to the samples, to a final volume of $25 \mu \mathrm{l}$.

\section{PCR and sequencing of target genes for comparative analyses}

Nested PCR was performed on DNA from single cells and trophozoites following the same protocols that have been used on DNA from crude isolates, generating a 530 bp amplicon of the tpi gene and a $511 \mathrm{bp}$ amplicon of the $b g$ gene $[22,23]$. Also, in order to verify the assemblages in the clinical samples as well as on all single cysts from isolate Sweh207, assemblage A and assemblage B specific PCRs for the tpi locus were performed [10,24].

PCR products were verified on $1.5 \%$ agarose gels stained with GelRed (Biotium, Hayward, CA, USA), proper amplicons were purified with Exo-SAP $\mathrm{IT}^{\mathrm{TM}}$ according to the manufacturer's instructions (GE Healthcare, Uppsala, Sweden) and sequenced bi-directionally using the BIG DYE 3.1 sequencing kit (Applied Biosystems, La Jolla, CA,
USA). Sequenced products were analyzed using the $A B$ 9100 sequence reader (Applied Biosystems, La Jolla, CA, USA), and subsequently examined and aligned utilizing the BioEdit software (Ver. 7.0.5.).

\section{Sequences}

The sequences generated in this study were submitted to GenBank, with the following accession numbers [GenBank: JN579665-JN579676] (tpi sequence set), and [GenBank: JN579677-JN579688] (bg sequence set).

\section{Results}

Isolation of Giardia cysts and trophozoites using micromanipulation

We have assessed the method of micromanipulation, using custom-made micro-capillaries synthesized with an inner diameter small enough to solely encapsulate single Giardia trophozoites or cysts, eliminating the risk of possible transfer of multiple cells. The clonality of all picked cells was further verified by microscopy, prior to transfer into the DNA extraction mixture. As a control, FITC-labeled cysts, purified from patient fecal material were transferred to 12 -well microscope slides $\left(n_{\text {tot }}=44\right.$ cysts) and fixed by desiccation, followed by the addition of mounting buffer to each well individually. The analysis was performed without the addition of cover slips in order to avoid cross contamination between the wells. The slides were analyzed using a fluorescence microscope and single cysts were present in all 44 wells. Also, all negative controls indicated the absence of Giardia cysts.

\section{Evaluation of different methods for DNA extraction and efficiency of PCR of single Giardia cells}

Two different methods were set up and evaluated in their efficiency of generating DNA from single trophozoites (GS/M-H7) that would yield sequences of high enough quality for the discrimination of ASH. PCR products could efficiently be produced using both protocols, however, the generation of sequences with double peaks in the expected positions showed complete efficiency only when applying the DNAreleasy protocol, as indicated in Table 1. Since the DNAreleasy protocol showed to be the most efficient for the extraction of high quality DNA from single trophozoites, it was subsequently also applied to the single cysts. Both the long and the short extraction protocols provided by the manufacturer were assayed. Applying the long extraction protocol yielded a higher number of positive results in subsequent PCR reactions (data not shown).

\section{Sequencing of Giardia from culture and at the single cell level}

Double peaks were stringently validated in the chromatograms of all sequences generated in this study. Only 
Table 1 Comparative sequence analysis of single GS/M trophozoites at the tpi locus

\begin{tabular}{|c|c|c|c|c|c|c|}
\hline \multirow[t]{2}{*}{ Isolate } & \multirow[t]{2}{*}{ Material } & \multirow[t]{2}{*}{ DNAreleasy } & \multirow[t]{2}{*}{ GenBank acc no } & \multicolumn{3}{|c|}{ Nucleotide position from start of gene } \\
\hline & & & & $39 *$ & 45 & 264 \\
\hline \multirow[t]{3}{*}{ GS/M } & Cloned sequence & & EF688030 & A & $\mathrm{T}$ & G \\
\hline & Cloned sequence & & EF688028 & G & C & A \\
\hline & Crude isolate & & FJ560571 & $\mathrm{R}$ & Y & $\mathrm{R}$ \\
\hline GS/M & Crude isolate & & $\mathrm{N} / \mathrm{A}$ & $\mathrm{R}$ & Y & $\mathrm{R}$ \\
\hline GS/M_3 & Single trophozoites & Not used & N/A & G & C & A \\
\hline GS/M_5 & & & & G & C & A \\
\hline GS/M_7 & & & & G & C & A \\
\hline GS/M_8 & Single trophozoite & Not used & N/A & A & $\mathrm{T}$ & G \\
\hline GS/M_6 & Single trophozoite & Not used & N/A & $\mathrm{R}$ & Y & $\mathrm{R}$ \\
\hline GS/M_71 & Single trophozoites & Used & JN579671 & $\mathrm{R}$ & Y & $\mathrm{R}$ \\
\hline GS/M_72 & & & & $\mathrm{R}$ & Y & $\mathrm{R}$ \\
\hline GS/M_73 & & & & $\mathrm{R}$ & Y & $\mathrm{R}$ \\
\hline GS/M_74 & & & & $\mathrm{R}$ & Y & $\mathrm{R}$ \\
\hline GS/M_76 & & & & $\mathrm{R}$ & Y & $\mathrm{R}$ \\
\hline GS/M_77 & & & & $\mathrm{R}$ & Y & $\mathrm{R}$ \\
\hline GS/M_78 & & & & $\mathrm{R}$ & Y & $\mathrm{R}$ \\
\hline GS/M_79 & & & & $\mathrm{R}$ & Y & $\mathrm{R}$ \\
\hline GS/M_80 & & & & $\mathrm{R}$ & Y & $\mathrm{R}$ \\
\hline
\end{tabular}

* This nucleotide position is a substitution pattern proposed as a marker for different B sub-assemblages [25].

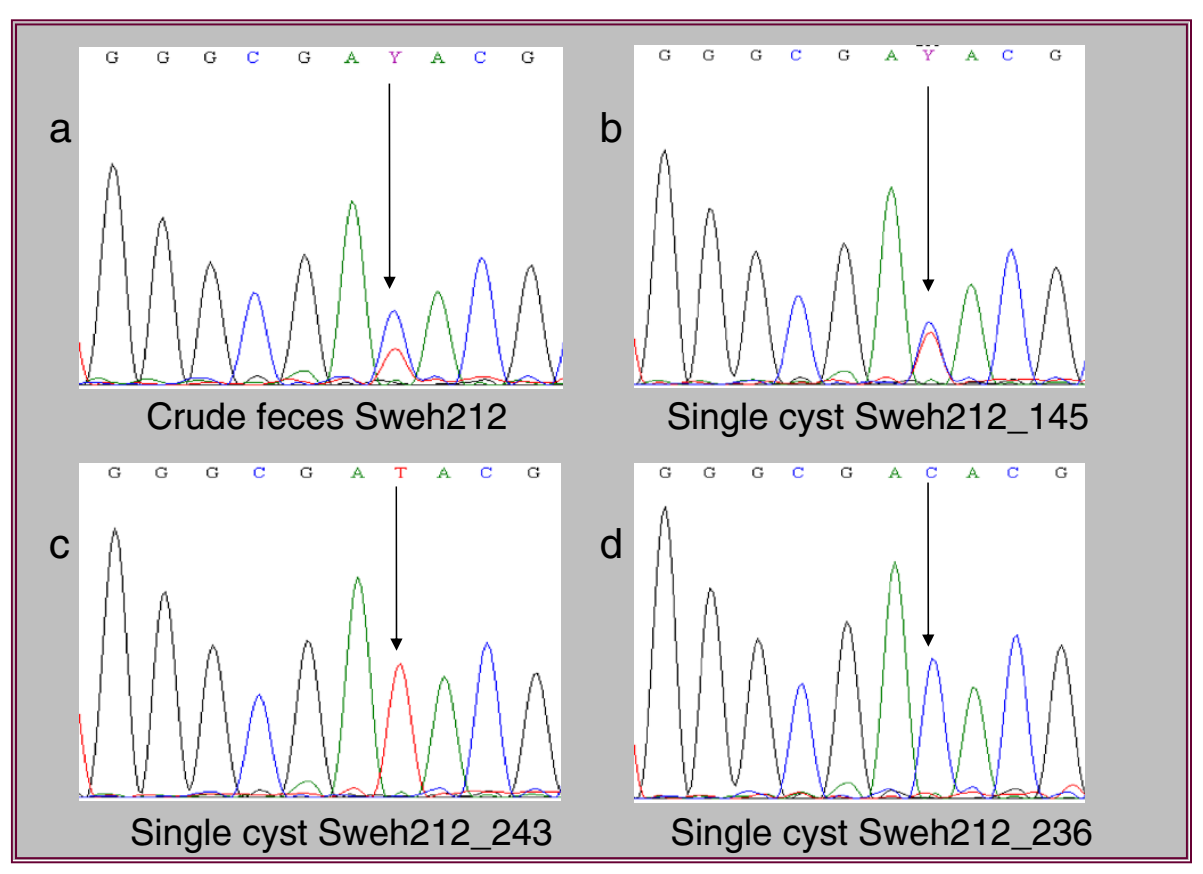

Figure 1 Sequence chromatograms of nucleotide variations. Chromatogram of a sequence generated from crude DNA from patient Sweh212, where the position indicated with an arrow shows the presence of a double peak (a). Sequencing of single cysts from the same patient indicates the presence of a double peak or ASH at the single cell level (b), and importantly, single cyst analyses also show that there are subpopulations present where double peaks do not exist in the same position (c and $\mathbf{d}$ ). 
chromatograms with no background bias were used, and positions in the chromatograms where double peaks were clearly visible were represented as $\mathrm{ASH}$ in the study. Figure 1, depicts one such position in Sweh212, where double peaks are present in sequences with DNA from crude feces, and single cyst Sweh212_145, but not in single cysts; Sweh212_243 or Sweh212_236 (Figure 1). Sequencing of the tpi locus generated from trophozoite cultures of the axenic, assemblage B isolate GS/M-H7, generated double peaks in three positions, namely 39,45 and 264 (Table 1) with the start codon set as position one. This sequence, along with sequences from public databases [GenBank: EF688030, EF688028 and FJ560571], were used as baseline for the GS/M-H7 analysis in order to define potential polymorphic subgroups when performing the single cell analyses (Table 1). Bi-directional sequencing of single GS/M-H7 trophozoites, with $(n=9)$ and without $(n=5)$ the pre-treatment of DNAreleasy, on a $530 \mathrm{bp}$ region of tpi was performed in order to verify the occurrence of ASH within single Giardia cells. The chromatograms were carefully analyzed with regards to double peaks, and forward and reverse sequences were subsequently aligned. All single GS/M-H7 trophozoites, which were pre-treated with DNAreleasy, displayed distinct double peaks in the same positions as those from the GS/M-H7 crude isolate (Table 1$)$. However, only one (20\%) of the single GS/MH7 trophozoites, that had not been pre-exposed to treatment with DNAreleasy, showed double peaks in all three positions (Table 1). Thus, DNAreleasy increases the amplification efficiency from single parasites.

$\mathrm{Bi}$-directional sequencing was also performed on DNA from clinical single cysts and sequences were aligned using variants of sub-assemblages BIII and BIV, as well as sequences from crude DNA from each respective sample as baselines, where possible. Positions that have earlier been suggested as variable between sub-assemblages BIII and BIV, are highlighted by an asterisk in Tables 1, 2, 3, 4, $5[10,25]$.

Sequencing of tpi PCR products from 13 cysts of patient isolate Sweh197 gave rise to six different sequence variants (Table 2). Only one sequence showed the same pattern as the crude isolate with double peaks at positions 39,114 , 165 and 280. The remaining sequences had either overlapping nucleotides at three positions or clean sequences without double peaks at any position (Table 2).

Beta-giardin sequences from six cysts, from sample Sweh212 gave rise to three different sequence variants (Table 3), where one variant indicated the same pattern as that of the crude DNA with double peaks in positions 369 and 516. The other two variants gave rise to sequences without any double peaks; one correlated with sub-assemblage BIV/Nij5 and [GenBank:HM165214] in positions 354, 369 and 516, and the other was identical to [GenBank:HM165216] (Table 3).

Cysts from isolate Sweh207 were investigated at two loci, $b g$ and tpi. Out of the cysts sequenced at the tpi locus, eight were assemblage $B$ and two were assemblage

Table 2 Comparative sequence analysis of single cysts from Sweh197 at the tpi locus

\begin{tabular}{|c|c|c|c|c|c|c|}
\hline \multirow[t]{2}{*}{ Sub-assemblagelsolate } & \multirow[t]{2}{*}{ Material } & \multirow[t]{2}{*}{ GenBank acc no } & \multicolumn{4}{|c|}{ Nucleotide position fromstart of gene } \\
\hline & & & $39 *$ & 114 & $165^{*}$ & 280 \\
\hline BIII/2924 & & AY228628 & G & C & C & A \\
\hline BIV/Ad-19 & & AF069560 & A & C & $\mathrm{T}$ & A \\
\hline Sweh197 & Crude stool isolate & JN579672 & $\mathrm{R}$ & Y & Y & $\mathrm{R}$ \\
\hline Sweh197_200 & Single cyst & JN579676 & $\mathrm{R}$ & Y & Y & $\mathrm{R}$ \\
\hline Sweh197_84 & Single cyst & JN579673 & $\mathrm{R}$ & C & Y & $\mathrm{R}$ \\
\hline Sweh197_201 & & & $\mathrm{R}$ & C & Y & $\mathrm{R}$ \\
\hline Sweh197_86 & Single cyst & JN579674 & $\mathrm{R}$ & Y & C & $\mathrm{R}$ \\
\hline Sweh197_98 & & & $\mathrm{R}$ & Y & C & $\mathrm{R}$ \\
\hline Sweh197_149 & & & $\mathrm{R}$ & Y & C & $\mathrm{R}$ \\
\hline Sweh197_213 & & & $\mathrm{R}$ & Y & C & $\mathrm{R}$ \\
\hline Sweh197_196 & Single cyst & JN579675 & A & C & C & G \\
\hline Sweh197_197 & & & A & C & C & G \\
\hline Sweh197_212 & & & A & C & C & G \\
\hline Sweh197_208 & & & A & C & C & G \\
\hline Sweh197_207 & Single cyst & EU272158 & G & $\mathrm{T}$ & C & A \\
\hline Sweh197_215 & Single cyst & GU56428 & G & C & $\mathrm{T}$ & A \\
\hline
\end{tabular}

* These nucleotide positions are substitution patterns proposed as markers for different B sub-assemblages [25]. 
Table 3 Comparative sequence analysis of single cysts from Sweh212 at the bg locus

\begin{tabular}{|c|c|c|c|c|c|}
\hline \multirow[t]{2}{*}{ Sub-assemblagelsolate } & \multirow[t]{2}{*}{ Material } & \multirow[t]{2}{*}{ GenBank acc no } & \multicolumn{3}{|c|}{ Nucleotide position from start of gene } \\
\hline & & & $354^{*}$ & 369 & 516 \\
\hline BIII/ BAH8 & & AY072727 & C & C & $\mathrm{T}$ \\
\hline BIV/ Nij5 & & AY072725 & $\mathrm{T}$ & C & $\mathrm{T}$ \\
\hline Sweh212 & Crude stool isolate & JN579687 & $T$ & $\mathrm{Y}$ & Y \\
\hline Sweh212_143 & Single cyst & JN579688 & $\mathrm{T}$ & Y & Y \\
\hline Sweh212_145 & & & $\mathrm{T}$ & Y & Y \\
\hline Sweh212_136 & Single cyst & HM165216 & $\mathrm{T}$ & $\mathrm{T}$ & C \\
\hline Sweh212_243 & & & $\mathrm{T}$ & $\mathrm{T}$ & C \\
\hline Sweh212_236 & Single cyst & HM165214 & $\mathrm{T}$ & C & $\mathrm{T}$ \\
\hline Sweh212_242 & & & $\mathrm{T}$ & C & $\mathrm{T}$ \\
\hline
\end{tabular}

* This nucleotide position is a substitution pattern proposed as a marker for different B sub-assemblages [10].

A. This was also verified using assemblage-specific nested PCR primers for tpi (data not shown). Sequences from the assemblage A parasites did not indicate any double peaks and corresponded to the sub-assemblage AII reference isolate, JH, [GenBank:U578978]. The eight assemblage $B$ sequences gave rise to five different variants at the tpi locus and polymorphisms were present in nine different positions (Table 4). One variant, including sequences from three cysts, was identical to the pattern seen in the crude isolate. Three of the variants had double peaks in two to four positions but lacked double peaks in certain positions compared to the pattern seen in the crude isolate, and one sequence was without double peaks. Sequences generated from crude DNA at the $b g$ locus from Sweh207 indicated the presence of both assemblage $A$ and $B$, therefore no crude DNA sequence is available for comparison at the $b g$ locus. However, bidirectional sequencing was performed on 15 single cysts, all of which were of the B assemblage.
Comparative analysis of the sequences yielded 11 different variants, and double peaks were present in at least one position in seven of the variants (Table 5).

\section{Discussion}

Giardia is a unique eukaryote where vegetative trophozoites, as far as we know, harbor two equal, diploid nuclei that contain five different chromosomes each [3]. The two nuclei, in the trophozoite, cycle between a diploid $(2 \mathrm{~N})$ and a tetraploid $(4 \mathrm{~N})$ genome content in the vegetative cell cycle. During the encystation process the DNA is replicated after cyst-wall formation, giving a cyst with a ploidy of $16 \mathrm{~N}$ in four nuclei [3]. The complex genetic makeup of this organism, in combination with published reports of high frequency of sequence polymorphisms in assemblage B Giardia [7,8,10,11], has raised the question of whether ASH occurs at the single cell level and how commonly multiple sub-assemblage infections occur in patients. Data from previously

Table 4 Comparative sequence analysis of single cysts from Sweh207 at the tpi locus

\begin{tabular}{|c|c|c|c|c|c|c|c|c|c|c|c|}
\hline \multirow{2}{*}{$\begin{array}{l}\text { Sub- } \\
\text { assemblagelsolate }\end{array}$} & \multirow[t]{2}{*}{ Material } & \multirow{2}{*}{$\begin{array}{l}\text { GenBank } \\
\text { acc no }\end{array}$} & \multicolumn{9}{|c|}{ Nucleotide position from start of gene } \\
\hline & & & $39 *$ & $91 *$ & 162 & $165^{*}$ & $168^{*}$ & 189 & $210^{*}$ & 258 & 423 \\
\hline BIII/ 2924 & & AY228628 & G & C & G & C & C & A & G & C & G \\
\hline BIV/Ad-19 & & AF069560 & A & T & G & T & T & A & A & C & G \\
\hline Sweh207 & Crude stool isolate & JN579665 & A & C & $\mathrm{R}$ & Y & $\mathrm{Y}$ & $\mathrm{R}$ & $\mathrm{R}$ & C & G \\
\hline Sweh207_161 & Single cyst & JN579666 & A & C & $\mathrm{R}$ & Y & Y & $\mathrm{R}$ & $\mathrm{R}$ & $C$ & G \\
\hline Sweh207_227 & & & A & C & $\mathrm{R}$ & Y & Y & $\mathrm{R}$ & $\mathrm{R}$ & C & G \\
\hline Sweh207_222 & & & A & $C$ & $\mathrm{R}$ & Y & Y & $\mathrm{R}$ & $\mathrm{R}$ & C & G \\
\hline Sweh207_166 & Single cyst & JN579667 & A & Y & $\mathrm{R}$ & Y & Y & A & $\mathrm{R}$ & C & $\mathrm{R}$ \\
\hline Sweh207_228 & & & A & C & $\mathrm{R}$ & Y & Y & $\mathrm{R}$ & $\mathrm{R}$ & C & G \\
\hline Sweh207_220 & Single cyst & JN579669 & $R$ & Y & R & Y & Y & A & $\mathrm{R}$ & C & R \\
\hline Sweh207_224 & Single cyst & JN579670 & $\mathrm{R}$ & Y & $\mathrm{R}$ & Y & Y & A & $\mathrm{R}$ & Y & R \\
\hline Sweh207_171 & Single cyst & JN579668 & $A$ & C & A & C & C & A & G & C & G \\
\hline
\end{tabular}

*These nucleotide positions are substitution patterns proposed as markers for different B sub-assemblages [25]. 
Table 5 Comparative sequence analysis of single cysts from Sweh207 at the bg locus

\begin{tabular}{|c|c|c|c|c|c|c|c|c|c|}
\hline \multirow[t]{2}{*}{ Sub-assemblage Isolate } & \multirow[t]{2}{*}{ Material } & \multirow[t]{2}{*}{ GenBank acc no } & \multicolumn{7}{|c|}{ Nucleotide position from start of gene } \\
\hline & & & 201 & 210 & 228 & 273 & 285 & $354^{*}$ & 537 \\
\hline BIII/BAH8 & & AY072727 & C & C & A & A & $\mathrm{T}$ & C & C \\
\hline BIV/Nij5 & & AY072725 & C & $\mathrm{T}$ & A & A & $\mathrm{T}$ & $\mathrm{T}$ & C \\
\hline Sweh207_65 & Single cyst & JN579677 & C & C & A & A & $\mathrm{T}$ & C & $\mathrm{T}$ \\
\hline Sweh207_66 & Single cyst & HM165209 & C & $\mathrm{T}$ & A & A & $\mathrm{T}$ & C & C \\
\hline Sweh207_133 & & & C & $\mathrm{T}$ & A & A & $\mathrm{T}$ & C & C \\
\hline Sweh207_103 & & & C & $T$ & A & A & T & C & C \\
\hline Sweh207_105 & Single cyst & AY072727 & C & C & A & A & $\mathrm{T}$ & C & C \\
\hline Sweh207_190 & Single cyst & JN579678 & C & C & A & G & $\mathrm{T}$ & C & C \\
\hline Sweh207_61 & Single cyst & JN579679 & C & C & A & $\mathrm{R}$ & T & C & C \\
\hline Sweh207_129 & Single cyst & JN579680 & C & $\mathrm{T}$ & $\mathrm{R}$ & A & $\mathrm{T}$ & C & C \\
\hline Sweh207_106 & Single cyst & JN579681 & C & C & $\mathrm{R}$ & A & T & Y & C \\
\hline Sweh207_107 & Single cyst & JN579682 & C & C & A & A & Y & C & C \\
\hline Sweh207_181 & & & C & C & A & A & Y & C & C \\
\hline Sweh207_184 & Single cyst & JN579683 & C & Y & A & A & $\mathrm{T}$ & C & Y \\
\hline Sweh207_186 & Single cyst & JN579684 & C & Y & A & $\mathrm{R}$ & T & C & C \\
\hline Sweh207_183 & Single cyst & JN579685 & Y & C & A & $\mathrm{R}$ & T & Y & C \\
\hline Sweh207_189 & Single cyst & JN579686 & Y & Y & $\mathrm{R}$ & $\mathrm{R}$ & $\mathrm{T}$ & Y & C \\
\hline
\end{tabular}

* This nucleotide position is a substitution pattern proposed as a marker for different B sub-assemblages [10].

published reports have indicated that ASH may occur at the single cell level $[6,12]$. However, these data are based on sequences generated at the population level from trophozoite cultures, and although there are strong indications of sequence divergence between the alleles, there has still been a necessity of verifying this at the single cell level in order to rule out any bias regarding potential mixed sub-genotypes in the trophozoite cultures. The previously analyzed isolates have been cloned using techniques that do not completely assure the source to be from a clonal cell line, and unintentional mixing of different in vitro cultures may cause cross-contamination problems when growing cells in microbiological laboratories. Thus, utilization of the micromanipulation technique rules out the risk of cross contamination since downstream analyses are performed on material from a single cell.

In order to validate allelic sequence divergence at the single cell level of G. intestinalis, it is of substantial importance to initiate the PCR reaction with high quality template DNA, where DNA from all alleles is present due to the complex nature of the assay. If the sensitivity of the analysis is not high enough, sequences produced in downstream reactions may indicate false negatives where regions of one or several alleles may not be properly amplified and would thereby not display double peaks in the chromatograms. The implementation of DNAreleasy showed full efficiency in the chromatograms produced from single trophozoites of the GS/M-H7 isolate. A freeze/thaw protocol, which was evaluated simultaneously also produced products in the nested PCR reaction in accordance with Miller et al [19]. This method however, only produced one sequence out of five that allowed the discrimination of double peaks in the chromatograms (Table 1). The in vitro establishment of viable assemblage B cysts (GS isolate) is highly inefficient (unpublished data), and therefore impeded the addition of a proper control for the purpose of verifying the presence of ASH in sequences generated from single Giardia cysts. DNAreleasy for DNA extraction was the only method sensitive enough to generate sequences where ASH could be detected when analyzing single trophozoites, therefore, two different DNAreleasy protocols provided by the manufacturer were assayed on the clinical cysts. Utilization of the long protocol indicated a higher proficiency in downstream PCR reactions (data not shown).

ASH was seen on the single cell level in all DNAreleasy treated single cells of the GS/M isolate, thus verifying our hypothesis of the occurrence of ASH in single Giardia assemblage $B$ parasites. Positions in the sequence on the tpi locus, that have earlier been highlighted as variable between different sub-assemblages or haplotypes of GS/M (Table 1) were here verified as double peaks, indicating sequence divergence in the different alleles in single Giardia cells. ASH also occurred at the single cell level in the majority of the cysts (21 out of 36 analyzed assemblage B cysts). However, the alignment of all sequences from a single patient sample did not result in the establishment of a 
consensus sequence. Instead, subgroups formed with unique single cysts or where sequences from two or more single cysts from the same sample origin aligned with complete sequence identity, but where the different subgroups or variants exhibited differences in the sequences in one or several positions. This further strengthens the concept of ASH at the single cell level and it also suggests that all three patients were infected with multiple subgenotypes of assemblage B Giardia. It is noteworthy, that since there are no reference sequences available for any of the cysts isolated from patients in this study it can not be ruled out that some of the sequence variants, where certain sequences do not indicate a mixed base at a position, could be due to a failure in detecting one of the alleles potentially present in a cyst where the DNA may be of suboptimal quality. Another factor, which could potentially influence misdetection of mixed bases is the possibility that some variant alleles may be present at a lower ratio than others and would thereby not be properly amplified and subsequently detected in the sequencing chromatogram (Tables 2, 3, 4 and 5). However, in Table 4, positions 39, 91, 258 and 423 indicate the presence of single nucleotides in the sequence from crude stool DNA, but sequences from several single cysts indicate mixed bases at one or several of these positions. Furthermore, many of the sequences from single cysts from the clinical samples indicated ASH at positions that have previously been suggested as variable for sub-assemblages BIII and BIV $[10,25]$. The common occurrence of ASH in these positions at the single cell level virtually renders these positions inept as discriminatory markers for sub-genotyping of assemblage B Giardia.

Sequences generated from single assemblage A and B cysts from patient Sweh207 at the tpi locus showed no indication of inter-assemblage recombination on the studied locus. However it would be of great interest to further analyze this on a larger population of samples harboring mixed assemblage $\mathrm{A}$ and $\mathrm{B}$ infection. The implementation of micromanipulation as an aid in verifying events of genetic exchange in Giardia would be highly beneficial. Sequencing based projects of specific target regions where potential recombination events are likely to occur, always include the risk that clinical samples may contain mixed sub-populations. Such bias would however, be completely eliminated if the sequencing was performed on a proficient number of single cysts isolated from populations of cysts from clinical samples using micromanipulation.

G. intestinalis has been assumed to be an asexual organism [26], but recent data suggest that this might not be true [27]. Epidemiological and population genetic studies have indicated recombination and allelic exchange between different Giardia isolates during infections [28]. Several meiosis-specific genes have been identified in the Giardia genome [29]. These genes have shown to be expressed during encystation, at the same time as fusion between the nuclei (diplomixis) has been detected [30]. ASH or single nucleotide polymorphisms have been detected in cultured Giardia isolates during genome sequencing projects $[12,31,32]$. However, considering the fact that the parasite has two diploid nuclei and the level of ASH is surprisingly low: $<0.01 \%$ in the sequenced assemblage A (WB) and E (P15) isolates and $0.5 \%$ in the assemblage $B$ (GS) isolate, it must mean that the parasites can actively reduce the level of $\mathrm{ASH}$ and that there must be some kind of communication between the two nuclei, as seen during the diplomixis process [30]. The striking differences in ASH levels between assemblage A and B isolates could imply that the different assemblages have different mechanisms in exchanging genetic material. Another possibility is that assemblage B isolates can fuse in a process similar to the newly discovered sexual process in Candida albicans and other pathogenic fungi [14]. In C. albicans, two diploid cells fuse and form a tetraploid cell that undergoes parasexual reduction to diploid or often aneuploid cells [14]. Aneuploid Giardia trophozoites have been reported [33], which could be remnants of cell fusion and reduction events. Thus, it is possible that the relatively high $\mathrm{ASH}$ levels in assemblage B $(0.5 \%)$ compared to assemblage A $(<0.01 \%)$ could be due to higher frequencies of cell fusions (sex) in assemblage B isolates. Yet another possibility is that the very low levels of ASH in assemblage A isolates could be due to highly active meiotic components, efficient diplomixis or efficient DNA repair systems.

Recent reports indicate that elevated levels of $\mathrm{ASH}$ in the pathogenic fungi, $C$. albicans, are linked to virulence and drug resistance [34,35]. Levert and colleagues have brought light to polymorphisms within bacterial populations and how this may be linked to the generation of virulence phenotypes, such as growth, resistance to stress or resistance to antibiotics [13]. Patient Sweh207, who had a mixed assemblage $A$ and $B$ infection, was subject to treatment failure. Interestingly, after treatment only the assemblage $\mathrm{B}$ parasites were present and sequencing indicated high levels of ASH both pre- and post- treatment in the assemblage B portion of the infection [8]. In the same study there were eight other reported cases of suspected treatment failure involving assemblage B infections, where sequencing of the parasites showed double peaks in several positions before and after treatment. Although this has to be further verified, the data brings forth a potential link between elevated levels of ASH and drug resistance in Giardia, as is the case in C. albicans.

\section{Conclusion}

We have developed a methodological pipeline that enables isolation and sequencing analyses of single $G$. 
intestinalis parasites. The presence of ASH was verified on the single cell level, both in cultured assemblage B trophozoites and in cysts from clinical samples. Also, the analysis of single cysts from patients made it apparent that one clinical, assemblage B containing sample, may often harbor the complexity of ASH in congruence with a mixed sub-assemblage infection. Additional file 2 is a schematic representation of the different possible outcomes in the event of an assemblage B Giardia infection. Moreover, the data presented here strongly highlights the necessity of re-evaluating the current molecular epidemiological methods used for sub-genotyping of assemblage B Giardia. The concurrence of ASH at the single cell level, and the seemingly high frequency of mixed sub-genotype infections in clinical samples makes it profoundly difficult to verify specific assemblage B sub-genotypes in clinical samples, using the current genotyping tools.

\section{Additional files}

Additional file 1: Single Giardia cells were isolated by micromanipulation, using micro capillaries with a $6-8 \mu \mathrm{m}$ inner diameter (panel A). Picked cells were transferred to a $2 \mu$ l pure drop of 1 X PBS for re-verification (panel B), and subsequently transferred to the PCR reaction mixture.

Additional file 2: A schematic representation of a mixed infection, where the red and blue bars represent different alleles of the same gene in different $G$. intestinalis sub-assemblages (a), and a single parasite harboring ASH, where red and blue bars indicate different alleles of the same gene within a single cell (b). This is a simplistic, schematic representation of different modes of infection in a giardiasis patient with parasites of different assemblage $B$ sub-assemblages, bringing forth the topics addressed in this study where mixed infection of different sub-assemblages, the occurrence of ASH in a clonal Giardia strain, or a mixture of the two may be present in a patient. Thus highlighting an important biological phenomenon in Giardia, as well as suggesting a revision of the current strategy used in assemblage $B$ Giardia epidemiology.

\section{Competing interests}

The authors declare that they have no competing interests.

\section{Acknowledgements}

This study was sponsored by grants from SIDA/SAREC, The Swedish Medical Research Council (VR-M) and Formas. We thank Görel Allestam for technical assistance. We also thank Professor Mats Wahlgren for generously providing us access to his micromanipulator.

\section{Author details}

${ }^{1}$ Department of Cell and Molecular Biology, BMC, Uppsala University, Husargatan 3, Box 596, Uppsala SE-751 24, Sweden. ${ }^{2}$ Department of Diagnostics and Vaccinology, Swedish Institute for Communicable Disease Control, Nobels väg 18, Solna SE-171 82, Sweden.

\section{Author's contribution}

$J A$ and $M L$ carried out the experiments and performed the data analyses. JA, $M L$ and SGS contributed to the design and coordination of the experiments. JA wrote the manuscript. ML and SGS participated in editing the manuscript. All authors have read and approved the manuscript.

Received: 01 December 2011 Accepted: 3 May 2012 Published: 3 May 2012

\section{References}

1. Lasek-Nesselquist E, Welch DM, Sogin ML: The identification of a new Giardia duodenalis assemblage in marine vertebrates and a preliminary analysis of $G$. duodenalis population biology in marine systems. Int $J$ Parasitol 2010, 40(9):1063-1074.

2. Ankarklev J, Jerlstrom-Hultqvist J, Ringqvist E, Troell K, Svard SG: Behind the smile: cell biology and disease mechanisms of Giardia species. Nat Rev Microbiol 2010, 8(6):413-422.

3. Bernander R, Palm JE, Svard SG: Genome ploidy in different stages of the Giardia lamblia life cycle. Cell Microbiol 2001, 3(1):55-62.

4. Caccio SM, Ryan U: Molecular epidemiology of giardiasis. Mol Biochem Parasitol 2008, 160(2):75-80.

5. Lebbad M, Ankarklev J, Tellez A, Leiva B, Andersson JO, Svard S: Dominance of Giardia assemblage B in Leon, Nicaragua. Acta Trop 2008, 106(1):44-53.

6. Wielinga C, Ryan U, Andrew Thompson RC, Monis P: Multi-locus analysis of Giardia duodenalis intra-Assemblage B substitution patterns in cloned culture isolates suggests sub-Assemblage $B$ analyses will require multilocus genotyping with conserved and variable genes. Int J Parasitol 2011, 41(5):495-503.

7. Bonhomme J, Le Goff L, Lemee V, Gargala G, Ballet JJ, Favennec L: Limitations of tpi and bg genes sub-genotyping for characterization of human Giardia duodenalis isolates. Parasitol Int 2011, 60(3):327-330.

8. Lebbad M, Petersson I, Karlsson L, Botero-Kleiven S, Andersson JO, Svenungsson B, Svard SG: Multilocus Genotyping of Human Giardia Isolates Suggests Limited Zoonotic Transmission and Association between Assemblage B and Flatulence in Children. PLoS Negl Trop Dis 2011, 5(8):e1262.

9. Cooper MA, Sterling CR, Gilman RH, Cama V, Ortega Y, Adam RD: Molecular analysis of household transmission of Giardia lamblia in a region of high endemicity in Peru. J Infect Dis 2010, 202(11):1713-1721.

10. Levecke B, Geldhof P, Claerebout E, Dorny P, Vercammen F, Caccio SM, Vercruysse J, Geurden T: Molecular characterisation of Giardia duodenalis in captive non-human primates reveals mixed assemblage A and B infections and novel polymorphisms. Int J Parasitol 2009, 39 (14):1595-1601.

11. Sprong H, Caccio SM, van der Giessen JW: Identification of zoonotic genotypes of Giardia duodenalis. PLoS Negl Trop Dis 2009, 3(12):e558.

12. Franzen O, Jerlstrom-Hultqvist J, Castro E, Sherwood E, Ankarklev J, Reiner DS, Palm D, Andersson JO, Andersson B, Svard SG: Draft genome sequencing of Giardia intestinalis assemblage B isolate GS: is human giardiasis caused by two different species?. PLoS Pathog 2009, 5(8):e1000560.

13. Levert M, Zamfir O, Clermont O, Bouvet O, Lespinats S, Hipeaux MC, Branger C, Picard B, Saint-Ruf C, Norel F, et al: Molecular and evolutionary bases of within-patient genotypic and phenotypic diversity in Escherichia coli extraintestinal infections. PLoS Pathog 2010, 6(9):e1001125.

14. Forche A, Alby K, Schaefer D, Johnson AD, Berman J, Bennett RJ: The parasexual cycle in Candida albicans provides an alternative pathway to meiosis for the formation of recombinant strains. PLOS Biol 2008, 6(5):e110.

15. Farnert A, Williams TN, Mwangi TW, Ehlin A, Fegan G, Macharia A, Lowe BS, Montgomery SM, Marsh K: Transmission-dependent tolerance to multiclonal Plasmodium falciparum infection. J Infect Dis 2009, 200(7):1166-1175.

16. Baum KF, Berens RL, Jones RH, Marr JJ: A new method for cloning Giardia lamblia, with a discussion of the statistical considerations of limiting dilution. J Parasitol 1988, 74(2):267-269.

17. Binz N, Thompson RC, Meloni BP, Lymbery AJ: A simple method for cloning Giardia duodenalis from cultures and fecal samples. J Parasitol 1991, 77(4):627-631.

18. Boreham PF, Phillips RE, Shepherd RW: Heterogeneity in the responses of clones of Giardia intestinalis to anti-giardial drugs. Trans R Soc Trop Med Hyg 1987, 81(3):406-407.

19. Miller KM, Sterling CR: Sensitivity of nested PCR in the detection of low numbers of Giardia lamblia cysts. Appl Environ Microbiol 2007, 73(18):59495950.

20. Chen Q, Barragan A, Fernandez V, Sundstrom A, Schlichtherle M, Sahlen A, Carlson J, Datta S, Wahlgren M: Identification of Plasmodium falciparum erythrocyte membrane protein 1 (PfEMP1) as the rosetting ligand of the malaria parasite $P$. falciparum. J Exp Med 1998, 187(1):15-23.

21. Brolin KJ, Ribacke U, Nilsson S, Ankarklev J, Moll K, Wahlgren M, Chen Q: Simultaneous transcription of duplicated var2csa gene copies in individual Plasmodium falciparum parasites. Genome Biol 2009, 10(10):R117.

22. Lalle M, Pozio E, Capelli G, Bruschi F, Crotti D, Caccio SM: Genetic heterogeneity at the beta-giardin locus among human and animal isolates of Giardia duodenalis and identification of potentially zoonotic subgenotypes. Int J Parasitol 2005, 35(2):207-213.

23. Sulaiman IM, Fayer R, Bern C, Gilman RH, Trout JM, Schantz PM, Das P, Lal AA, Xiao $\mathrm{L}$ : Triosephosphate isomerase gene characterization and potential 
zoonotic transmission of Giardia duodenalis. Emerg Infect Dis 2003, 9 (11):1444-1452.

24. Geurden T, Geldhof P, Levecke B, Martens C, Berkvens D, Casaert S, Vercruysse J, Claerebout E: Mixed Giardia duodenalis assemblage A and E infections in calves. Int J Parasitol 2008, 38(2):259-264.

25. Wielinga CM, Thompson RC: Comparative evaluation of Giardia duodenalis sequence data. Parasitology 2007, 134(Pt 12):1795-1821.

26. Tibayrenc M, Kjellberg F, Ayala FJ: A clonal theory of parasitic protozoa: the population structures of Entamoeba, Giardia, Leishmania, Naegleria, Plasmodium, Trichomonas, and Trypanosoma and their medical and taxonomical consequences. Proc Natl Acad Sci U S A 1990, 87(7):2414-2418.

27. Birky CW Jr: Giardia sex? Yes, but how and how much?. Trends Parasitol 2010, 26(2):70-74.

28. Cooper MA, Adam RD, Worobey M, Sterling CR: Population genetics provides evidence for recombination in Giardia. Curr Biol 2007, 17 (22):1984-1988.

29. Ramesh MA, Malik SB, Logsdon JM Jr: A phylogenomic inventory of meiotic genes; evidence for sex in Giardia and an early eukaryotic origin of meiosis. Curr Biol 2005, 15(2):185-191.

30. Poxleitner MK, Carpenter ML, Mancuso JJ, Wang CJ, Dawson SC, Cande WZ Evidence for karyogamy and exchange of genetic material in the binucleate intestinal parasite Giardia intestinalis. Science 2008, 319 (5869):1530-1533.

31. Jerlstrom-Hultqvist J, Franzen O, Ankarklev J, Xu F, Nohynkova E, Andersson JO, Svard SG, Andersson B: Genome analysis and comparative genomics of a Giardia intestinalis assemblage E isolate. BMC Genomics 2010, 11:543.

32. Morrison HG, McArthur AG, Gillin FD, Aley SB, Adam RD, Olsen GJ, Best AA, Cande WZ, Chen F, Cipriano MJ, et al: Genomic minimalism in the early diverging intestinal parasite Giardia lamblia. Science 2007, 317(5846):1921-1926

33. Tumova P, Hofstetrova K, Nohynkova E, Hovorka O, Kral J: Cytogenetic evidence for diversity of two nuclei within a single diplomonad cell of Giardia. Chromosoma 2007, 116(1):65-78.

34. Selmecki A, Forche A, Berman J: Aneuploidy and isochromosome formation in drug-resistant Candida albicans. Science 2006, 313(5785):367-370

35. Alby K, Bennett RJ: Sexual reproduction in the Candida clade: cryptic cycles, diverse mechanisms, and alternative functions. Cell Mol Life Sci 2010, 67(19):3275-3285.

doi:10.1186/1471-2180-12-65

Cite this article as: Ankarlev et al: Allelic sequence heterozygosity in single Giardia parasites. BMC Microbiology 2012 12:65.

\section{Submit your next manuscript to BioMed Central and take full advantage of:}

- Convenient online submission

- Thorough peer review

- No space constraints or color figure charges

- Immediate publication on acceptance

- Inclusion in PubMed, CAS, Scopus and Google Scholar

- Research which is freely available for redistribution

Submit your manuscript at www.biomedcentral.com/submit
(O) Biomed Central 